\title{
Avaliação clínica e histopatológica do bulbo do olho de cães, após aplicação de flunixin meglumine por via subconjuntival*
}

\author{
Clinical and histopathological analisis of the dog's ocular tissues, \\ after flunixin meglumine subconjunctival injection
}

Paula Diniz Galera, ${ }^{* * * * *}$ José Luiz Laus, ${ }^{* *}$ Cássio Ricardo Ribeiro,**,*** Ana Maria Barros Soares ${ }^{* * * * * *}$

\begin{abstract}
Resumo
Frente à eficácia do uso de flunixin meglumine nas inflamações oculares em cães, pelas vias oral, intravenosa, intramuscular e subcutânea, concebeu-se testar a via de administração subconjuntival, para uso oftálmico, nesta espécie e os efeitos deste fármaco sobre os tecidos oculares. Animais da espécie canina, adultos, machos e fêmeas, clinicamente sadios receberam, por aplicação subconjuntival, flunixin meglumine na dose de $1,1 \mathrm{mg} / \mathrm{kg}$ em olho direito, durante três dias consecutivos. O olho contralateral (controle) recebeu solução salina a $0,9 \%$ em volume idêntico ao do fármaco. Estudaram-se as eventuais alterações sobre o bulbo do olho mediante exames clínico e histopatológico. Os resultados obtidos mostraram blefarospasmo, hiperemia conjuntival e quemose transitórias no sítio de aplicação e blefarospasmo. Os bulbos oculares foram coletados ao término do protocolo terapêutico. A histopatologia dos olhos submetidos à aplicação do flunixin meglumine revelou infiltrado celular moderado, sob a conjuntiva, em olhos direito de cinco animais e leve em olho esquerdo de um cão. Pode-se observar a manutenção da integridade do segmento posterior do olho em todos os animais, mormente pela coesão das três túnicas oculares.
\end{abstract}

Palavras-chave: flunixin meglumine, olho, cão, aplicação subconjuntival, inflamação

\begin{abstract}
Considering the good theraupeutic effect of flunixin meglumine by oral, intravenous, intramuscular and subcutaneous routeson canine ophtalmic inflammation, we decided to evaluate the effect of subconjunctival injections of the drug. Healthy adults dogs (males and females) were selected to receive daily subconjunctival injections for three consecutive days of $1.1 \mathrm{mg} / \mathrm{kg}$ of flunixin meglumine on the right eye and the same volume of saline on the left eye. Local toxicity was assessed by clinical exam and histopathology. Ocular unrest with blefarospasm and conjunctival hiperemia on the site of injection with edema of the subconjuctival bubble was observed. The eyes were collected at the end of therapeutic protocol. The histopathology of five right eyes showed moderated cellular infiltrate under the conjunctive, and one left eye showed mild cellular infiltrate. Posterior segment integrity was maintained in all animals.
\end{abstract}

Keywords: Flunixin meglumine, eye, subconjunctival injection, infammation, dog.

\section{Introdução}

Entende-se por inflamação a resposta natural do organismo frente à injúria (Cotran et al., 1999). No escopo de defendê-lo de estímulos nocivos externos, a resposta inflamatória, de per se, implica alteração tecidual local, com danos decorrentes. A inflamação ocular é, particularmente, nociva por se manifestar de forma abrupta, interferindo com a visão, quando persistente (Peiffer, 1980). Sem o seu controle efetivo, pode induzir a alterações estruturais (Conlon, 1988), como sinéquias e aumento da permeabilidade vascular, seguidas de modificações na pressão intra-ocular (Boothe, 1984) e eventual perda da visão (Hall E Bonta, 1977; Shiels et al., 1999). Uveíte é condição comum em muitos dos processos mórbidos intra-oculares, com uma ampla variedade de causas exógenas e endógenas que resultam, em sua maioria, na liberação de mediadores químicos provenientes do metabolismo do ácido araquidônico (Higgins e Lees, 1984; Dziezyc, 1989; Millichamp et al., 1991).

A compreensão dos mecanismos e efeitos da inflamação ocular é condição sine qua non para o uso correto de antiinflamatórios esteroidais e não-esteroidais. Desde que a causa suscitante, em um determinado paciente, é de difícil

\footnotetext{
*Parte de tese de doutorado, do programa de pós-graduação em Cirurgia Veterinária da FCAV/UNESP/Jaboticabal, SP.

** Faculdade de Ciências Agrárias e Veterinárias - UNESP/Jaboticabal, SP

*** União Pioneira de Integração Social/UPIS - Brasília,DF; pgalera@terra.com.br

${ }^{* * * *}$ Faculdade de Medicina Veterinária - Universidade Federal Fluminense - UFF /Niterói, RJ.
} 
identificação, o tratamento da uveíte fundamenta-se, em primeira instância, na atenuação da inflamação ocular (Shiels et al., 1999). Substâncias que inibem a síntese, liberação ou ação das prostaglandinas no olho auxiliam no controle da inflamação ocular (Peiffer, 1980; Krohne e Vestre, 1987a; Khrone e Vestre, 1987b). Prostaglandinas encontradas em áreas inflamadas, inicialmente, advêm de tecidos lesados, desestabilizando a barreira hemato-aquosa. Antiinflamatórios não-esteroidais bloqueiam a síntese de prostaglandinas (Hall \& Bonta, 1977; Boothe, 1989; Regnier, 1999; Forsyth et al., 2000). O flunixin meglumine, potente agente não-esteroidal com atividade analgésica, antiinflamatória e antipirética, bloqueia a produção de prostaglandinas por meio de inibição das ciclooxigenases (Bistner et al., 1996; Shiels et al., 1999).

O uso oftálmico de antiinflamatórios não-esteroidais inclui indicações terapêuticas e profiláticas. O flunixin meglumine, um derivado do ácido nicotínico (Colon, 1988), tem-se mostrado efetivo no controle da inflamação ocular (Tasaka, 2000). Inibição da neoformação vascular corneana (Hendrix, 1995), controle da uveíte, mesmo aquela decorrente de procedimentos cirúrgicos (Colon, 1988; Krohne e Vestre, 1989; Collins e Moore, 1999; Mathews, 2000; Laus et al., 2001), atuação sobre a manutenção da pressão intra-ocular (Shiels et al., 1999), bem como a redução da concentração de prostaglandinas no humor aquoso (Millichamp et al., 1991; Millichamp e Dziezyc, 1991), são efeitos desejáveis deste fármaco.

Antiinflamatórios não-esteroidais administrados sistemicamente geram efeitos colaterais indesejáveis, como intolerância e hemorragias gastrintestinais (Boothe, 1989; King e Miller, 1997), disfunções renais (Elwood et al., 1992; Mcneil, 1992; Lobetti e Joubert, 2000) e injúria hepática (Krohne e Vestre, 1989), o que impõe restrições à sua utilização.

A terapia ocular oferece oportunidades de tratamento únicas, pois é um do poucos sistemas orgânicos de rápido acesso terapêutico e monitoramento (Mealey, 2000). A farmacocinética na aplicação tópica permite a maximização da concentração do medicamento no sítio de ação desejado, enquanto minimiza sua concentração em outros tecidos orgânicos, onde ocorreriam os efeitos adversos (Mealey, 2000). Conhecendose as propriedades farmacológicas, o local de ação, a freqüência de administração e a concentração, pode-se determinar a melhor via de administração de um fármaco (Slatter, 2001).

Medicamentos com finalidade de terapia oftálmica, se administrados por via sistêmica, podem ter limitadas suas ações, em face da pobre penetração nos tecidos oculares, constituindo-se, apenas, em terapia adjutória. Dentre as rotas de distribuição local, as injeções subconjuntivais e intravítreas são cada vez mais indicadas. Não obstante, deve-se considerar as limitações destes acessos, que podem desencadear distúrbios aos tecidos oculares (Mathis, 1999). Injeções subconjuntivais, de maneira geral, são bem toleradas quando não mais que $1 \mathrm{~mL}$ de líquido é injetado (Slatter, 2001). A absorção de medicamentos pelo bulbo do olho, via escleral, é bastante significativa e, em muitos casos, de duração relativamente prolongada. A via de aplicação subconjuntival implica injeção da substância sob a conjuntiva, proximamente ao limbo. A partir da bolha formada, a substância difunde-se para o bulbo do olho ou, diretamente, para a câmara anterior (Mathis, 1999; Slatter, 2001) e, ainda, para o filme lacrimal (Ward e Clark, 1991).
Frente à eficácia terapêutica do flunixin meglumine nas inflamações oculares, mormente nas ceratites e nas uveítes, bem como nos traumas diversos, e à sua utilização e corrente indicação na profilaxia da inflamação em cirurgias intra-oculares, concebeu-se testar, em cães, a via de administração subconjuntival para o fármaco, cujos efeitos são desconhecidos. Entende-se que concentrações locais mais elevadas seriam atingidas, aliada à pouca exposição dos outros tecidos orgânicos à substância. Para tanto, considerou-se a necessidade da avaliação de eventuais efeitos adversos sobre os tecidos oculares.

\section{Material e método}

\section{Animais}

Foram utilizados dez animais da espécie canina, sem raça definida, machos ou fêmeas, adultos, com peso médio de $10 \mathrm{~kg}$ (entre 8 e $12 \mathrm{~kg}$ ). Os animais foram submetidos à avaliação clínica, à hematologia e à bioquímica sérica (alanina amino-transferase, aspartato amino-transferase, uréia, creatinina e gama glutamil transferase) e a exame oftálmico rotineiro, para a constatação das condições de higidez orgânica. Foram, ainda, mantidos em canis individuais apropriados, ${ }^{1}$ com alimentação adequada ${ }^{2}$ e água ad libitum.

\section{Protocolo terapêutico}

Aos animais administrou-se flunixin meglumine ${ }^{3}$ na dose de $1,1 \mathrm{mg} / \mathrm{kg}$ de peso corpóreo, por via subconjuntival, em conjuntiva bulbar temporal superior, no olho direito, a cada 24 horas, durante três dias consecutivos (Figura 1A,C,D), não excedendo $0,5 \mathrm{~mL}$ de volume final. $\mathrm{O}$ olho contralateral constituiu-se em controle, recebendo igual volume de solução salina estéril ${ }^{4}$ (Figura $1 \mathrm{~B}$ ). Utilizou-se seringa ${ }^{5}$ de $1,0 \mathrm{~mL}$ acoplada a agulha hipodérmica ${ }^{6}$ para as injeções.

Houve a colaboração de todos os cães durante os três dias de aplicação, pois se mostraram extremamente dóceis, uma vez que foram mantidos sob "quarentena" previamente à realização do experimento e tratados por uma mesma pessoa.

Durante a manipulação os animais não foram submetidos à condição de estresse, porquanto não houve a necessidade de contenção química ou mesmo a utilização de mordaça. Foram manipulados sentados, sobre uma mesa de procedimento, seguros pelo focinho e tranqüilizados pela voz da pessoa responsável pelo experimento.

\footnotetext{
dimensões 3,0 ×2,0 m área coberta e solarium com 4,5 x 2,0 m (área total: $15 \mathrm{~m} 2$ )

2 Special Croc - Royal Canin SA - Descalvado, SP

3 Banamine injetável - Schering-Plough-Coopers - São Paulo, SP

4 Solução Salina a 0,9\% - JP Industria Farmacêutica - Ribeirão Preto, $\mathrm{SP}$

5 Seringa de $1 \mathrm{~mL}$ - Becton \& Dickinson Industrias Cirúrgicas - Juiz de Fora, MG

6 Agulha Hipodérmica 13 × 4,5 - Becton \& Dickinson Industrias Cirúrgi-
} cas - Juiz de Fora, MG 


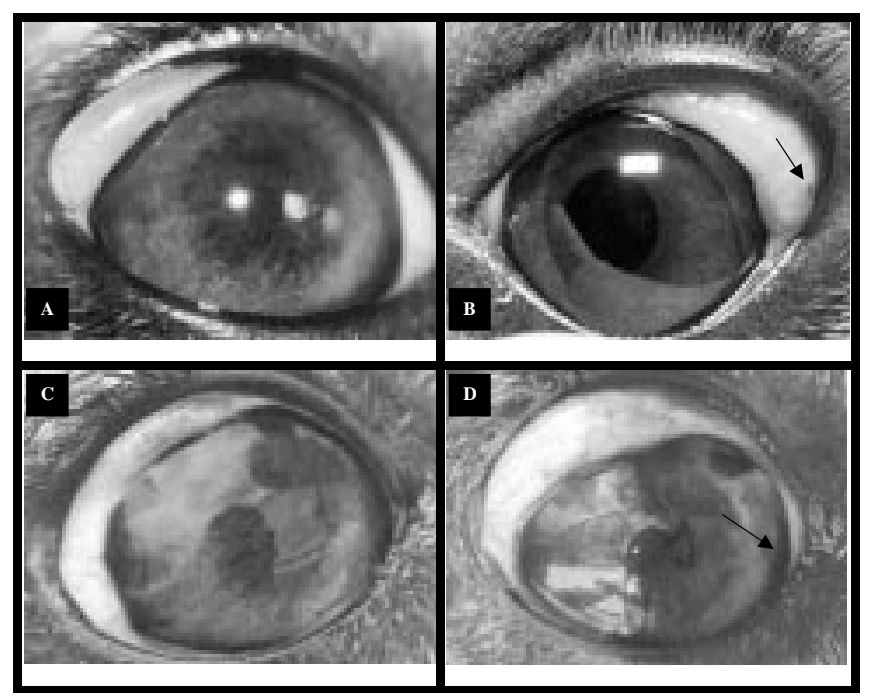

Figura 1: Imagem fotográfica de olho direito (A) e esquerdo (B) de cão, ilustrando a formação de uma bolha após injeção subconjuntival de flunixin meglumine e solução salina estéril a $0,9 \%$, respectivamente, ao 10 dia de aplicação. Observar ponto hemorrágico no local de aplicação (B) (seta). Imagem fotográfica de olho direito de cão, após injeção subconjuntival de flunixin meglumine, ao $2 \circ$ (C) e $3 \circ$ (D) dia de aplicação.

\section{Protocolos de Avaliação}

\section{Avaliação oftálmica}

Avaliaram-se as condições locais no sítio da injeção subconjuntival do fármaco e aquelas referentes aos sinais de desconforto ocular como blefarospasmo, hiperemia conjuntival, quemose, secreção e condições de transparência da córnea. Empregou-se método subjetivo, quali-quantitativo, com as seguintes intensidades indicadas por cruzes: leve $(+)$, moderado $(++)$ e intenso $(+++)$.

\section{Histopatologia}

Decorridas 24 horas do terceiro dia da aplicação subconjuntival do flunixin meglumine, os animais foram submetidos a medicação pré-anestésica com levomepromazina, ${ }^{7}$ na dose de $1,0 \mathrm{mg} / \mathrm{kg}$, e anestesia geral com tiopental sódico, ${ }^{8}$ por via intravenosa, na dose média de $12,5 \mathrm{mg} / \mathrm{kg}$ (Fantoni et al., 2000), em bolus. Enuclearam-se, por técnica transpalpebral (Slatter, 2001), os globos oculares direito e esquerdo para anatomopatologia. O material coletado foi imerso em formalina tamponada a $10 \%$, em solução aquosa para, decorridos os tempos necessários à fixação, ser incluído em parafina, cortado (cortes seriados) em micrótomo $(5 \mathrm{~mm})$ e corado pela hematoxilina-eosina (HE) e avaliado à microscopia óptica.
Ressalvas cabem ao destino destes animais, que eram provenientes do Centro de Zoonoses e ficara previamente estabelecido que os mesmos seriam utilizados para ensino de anatomia, razão pela qual foram levados a óbito, o que se deu por injeção intravenosa de cloreto de potássio, ${ }^{9}$ quando ainda se encontravam sob anestesia geral.

\section{Resultados}

\section{Avaliação oftálmica}

A avaliação oftálmica rotineira dos olhos submetidos à ação do flunixin meglumine, aplicado por via subconjuntival, permitiu que se constatasse desconforto ocular discreto ao primeiro dia após a injeção em todos os animais, manifesto por blefarospasmo imediato à aplicação do medicamento. Entretanto, a ocorrência se deu de forma fugaz, desaparecendo decorridos poucos minutos. Secreção ocular seromucosa foi observada, em grau leve (+), em dois animais, ao terceiro dia de aplicação. Ainda quanto às intercorrências no local da injeção, no segundo e terceiro dias pode-se observar, em todos os animais, hiperemia no ponto de aplicação e alguma quemose, de graus leve (+) a moderado (++).

No terceiro dia havia, ainda, a não-reabsorção completa do medicamento em três animais, caracterizada pela persistência de parte da bolha subconjuntival e adelgaçamento da conjuntiva bulbar. Em dois cães, devido ao adelgaçamento subconjuntival, houve extravasamento do fármaco por sobre a córnea por ocasião da aplicação, o que gerou edema corneano moderado $(++)$.

Observou-se, nos olhos controle, diminuição rápida da bolha subconjuntival no curso dos dias de aplicação. Houve reabsorção da solução salina estéril com algumas variações temporais individuais. Blefarospasmo manifestou-se, apenas, no momento da manipulação oftálmica, por ocasião da injeção. Não se constataram hiperemia, tampouco quemose ou quaisquer outras intercorrências.

\section{Histopatologia}

A microscopia óptica revelou infiltrado celular, caracterizado pela presença de neutrófilos e linfócitos, de forma moderada $(++)$, sob a conjuntiva, em olho direito (Figura 2 ; 3) de cinco animais, e de forma leve (+) em olho controle de um cão. Observou-se ceratite em um cão, decorrente do extravasamento do fármaco por sobre a córnea, caracterizada por infiltrado intenso de polimorfonucleares, mormente linfócitos em estroma corneano. Foram observados vacúolos no corpo ciliar em olhos direito e esquerdo de dois cães. Pode-se observar a manutenção da integridade do segmento posterior do olho em todos os animais, mormente pela coesão das três túnicas oculares (Figura 4).

\footnotetext{
7 Neozine - Aventis Pharma - São Paulo, SP.

8 Tiopental sódico - Cristália - Prod. Quim. Farm. Ltda. - São Paulo, SP.
}

9 Cloreto de Potássio a 19,1\% - Equiplex Ind. Quim \& Farm. Ltda Aparecida de Goiânia, GO. 


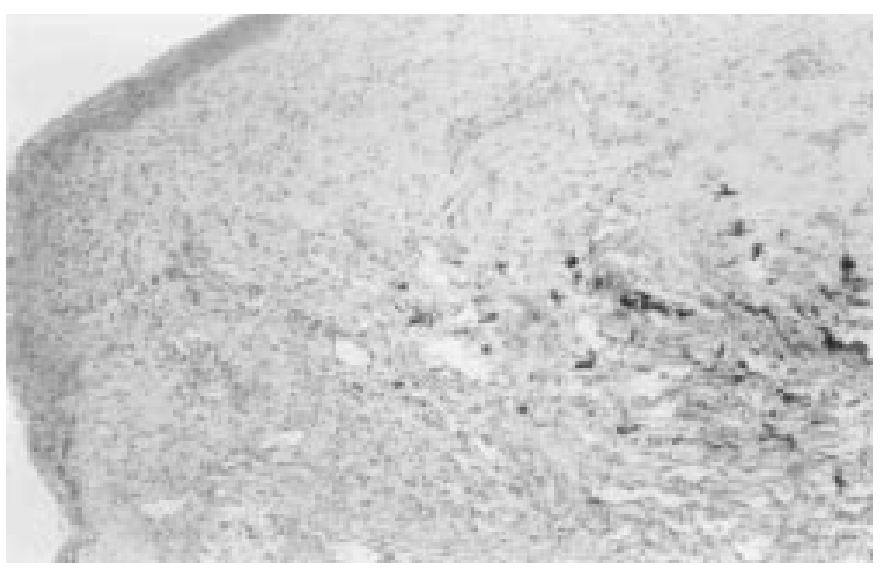

Figura 2 - Imagem fotomicrográfica de corte conjuntiva bulbar de cão submetido à aplicação subconjuntival de flunixin meglumine (Banamineâ) durante três dias consecutivos, a intervalos de 24 horas. (10X10). Observar maior celularidade na região inferior.

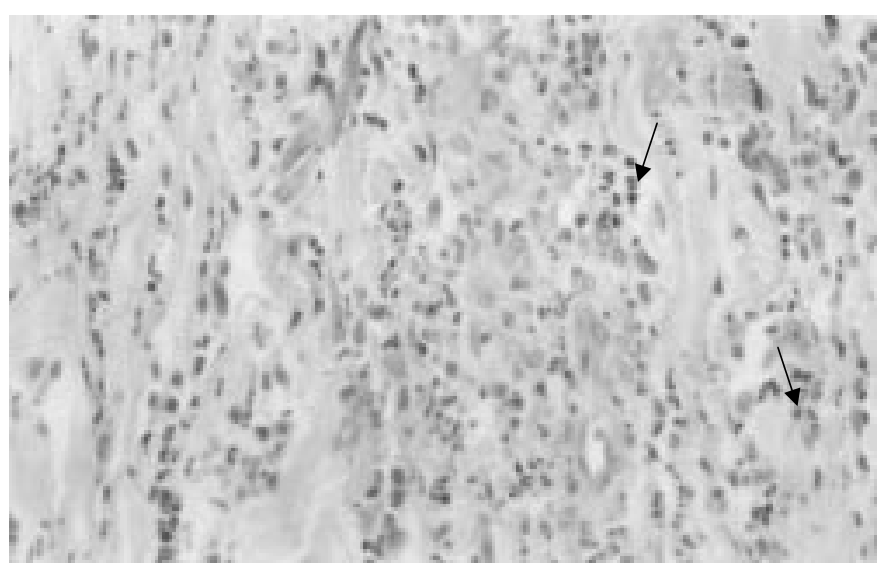

Figura 3 - Imagem fotomicrográfica de corte de tecido subconjuntival de olho de cão submetido à aplicação subconjuntival de flunixin meglumine (Banamineâ), na dose de $1,1 \mathrm{mg} / \mathrm{kg}$, por três dias consecutivos, a intervalos de 24 horas. $(20 \times 10)$. Aumento da Figura 2. Observar infiltrado polimorfonuclear moderado (setas).

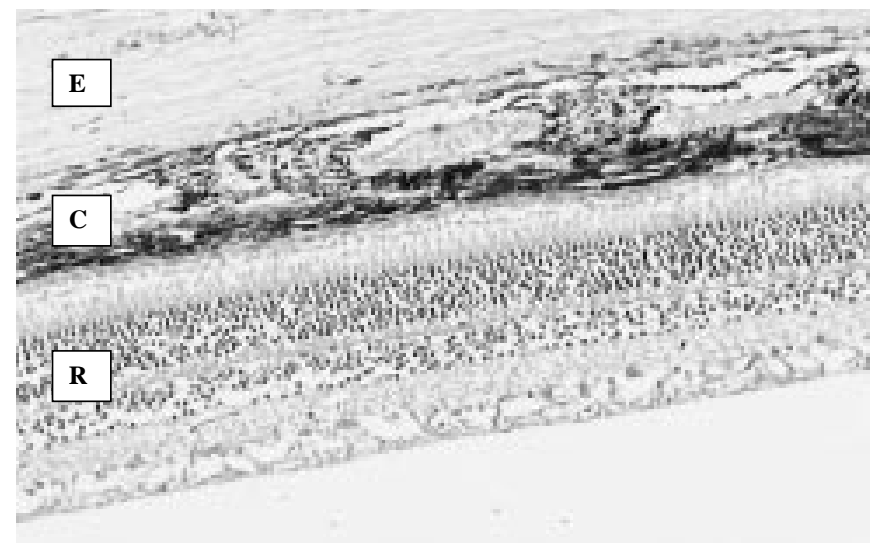

Figura 4: Imagem fotomicrográfica de corte das túnicas oculares (esclera $E$, coróide $C$ e retina $R$ ) de olho de cão submetido à aplicação subconjuntival de flunixin meglumine(Banamineâ), na dose de $1,1 \mathrm{mg} /$ $\mathrm{kg}$, por três dias consecutivos, a intervalos de 24 horas. (10X10). Observar a integridade e coesão das estruturas, sem sinais de inflamação.

\section{Discussão}

Consideram-se únicas as oportunidades viáveis de tratamento em oftalmologia (Mealey, 2000). A injeção subconjuntival foi considerada pensando-se na necessidade em se manter por tempo prolongado os efeitos de medicamentos implicando em menor freqüência de administração e, conseqüentemente, em menor custo (Blair et al., 1999). Outrossim, em atenção à tendência crescente quanto à utilização de substâncias por via local (Mealey, 2000), e por ser a absorção medicamentosa pelo bulbo do olho, mediante a via escleral, bastante efetiva (Collins e Moore, 1999).

De consenso são os efeitos colaterais, à distância, resultantes do emprego de antiinflamatórios não-esteroidais (Vonderhaar e Salisbury, 1993; Mathews et al., 1996; King e Miller, 1997; Cruz et al., 1998), o que obriga ponderar seu uso sob certas condições.

Os resultados obtidos permitiram constatar exsudação inflamatória aguda sob a conjuntiva, em cinco dos olhos tratados com o flunixin meglumine e em um olho mantido como controle. Dado que, por definição, a inflamação é uma resposta natural do tecido vivo a uma injúria (Cotran et al., 1999), atribui-se que os achados deram-se em decorrência da manipulação ocular e da injeção subconjuntival. Hiperemia conjuntival, quemose, descarga ocular, fotofobia e blefarospasmo são reações imediatas à ação de trauma ou de substâncias nocivas (Millichamp e Diziezyc, 1991). Relativamente aos olhos tratados, há que se considerar, ainda, a ação da solução alcalina que compõe o fármaco (Boothe, 1989). Substâncias alcalinas são de irritação tecidual que, não raro, resultam em infiltração perivascular e moderada a intensa perivasculite. Assim é que poucos AINES são factíveis para uso intravenoso (Boothe, 1989). Ao contrário dos cães, em que não há informações definitivas, em eqüinos o medicamento não é bem tolerado pela via subconjuntival (Lees e Higgins, 1984) e, por isso, não deve ser empregado (Stades et al., 1999).

Atribui-se, ainda, à exsudação inflamatória, a concentração do fármaco no sítio de injeção, significativamente elevada, já que aplicações locais possuem tal característica (Mathis, 1999). Admitiu-se, para esta aplicação, a mesma dose proposta para administrações por via oral, subcutânea ou intravenosa (Hardie et al., 1985). Aplicações locais favorecem a formação de um depósito. Na injeção subconjuntival dá-se a formação de uma bolha, a partir da qual a liberação da substância tende a ser lenta e gradual, substituindo aplicações repetidas.

Conceberam-se três administrações consecutivas do flunixin meglumine visando-se a avaliação dos efeitos colaterais em dosagem mais elevada, transcendendo-se o habitual. Segundo Mealey (2000), a farmacocinética tópica maximiza a concentração da substância no sítio de ação desejado. Expôs-se, portanto, o tecido ocular a maiores concentrações do fármaco, se comparado ao que seria habitualmente proposto para esta via de aplicação. Propôs-se que não mais que $0,5 \mathrm{~mL}$ fosse injetado, de consoante com Stades et al. (1999) e Slatter (2001).

A via de aplicação subconjuntival permite que a substância difunda-se para outras estruturas oculares (Slatter, 1990; Mathis, 1999). Entretanto, não se verificaram alterações em tecidos oculares outros que não naqueles próximos ao sítio 
de aplicação. A degeneração observada no corpo ciliar de dois animais, através da formação de cistos, não parece ter relação com o fármaco, porquanto se encontrava presente nos dois olhos.

O flunixin meglumine injetável, à concentração de $5 \%$, ou o seu veículo, provavelmente acarreta certa toxicidade para a córnea na forma de "colírio", haja vista a exsudação inflamatória que determinou naqueles olhos em que se deu o seu extravasamento acidental para a superfície corneana, sabidamente sensível à ação dos álcalis (Donzis et al., 1987; Wagoner, 1997). Considerando-se que a injeção subconjuntival permite a difusão de substâncias para a coróide e retina (Mathis, 1999), não se observaram alterações que possibilitem falar da ação tóxica sobre estes tecidos.

O interesse crescente pelo emprego de antiinflamatórios nãoesteroidais na oftalmologia veterinária vem suscitando, cada vez mais, pesquisas esclarecedoras, relativamente a um campo que merece ser melhor explorado. Dadas as particularidades do órgão da visão, quanto à sua fisiologia e metabolismo (Millichamp e Dziezyc, 1991), parece oportuno e com- pulsório estudarem-se e esclarecerem-se questões ainda controversas, quanto à via subconjuntival e à ação de substâncias.

\section{Conclusões}

Frente às condições experimentais concebidas, permite-se admitir que:

- a aplicação de flunixin meglumine (Banamineâ), em dose única, não acarretou alterações macroscópicas no ponto de injeção;

- em função de alterações oculares locais, o uso de flunixin meglumine (Banamine ${ }^{\hat{a}}$ ) em doses consecutivas deve ser feito com cautela ou mesmo evitado;

- a aplicação do fármaco em doses consecutivas resultou em exsudação inflamatória observada à microscopia óptica no ponto de aplicação;

- a aplicação do fármaco em doses consecutivas não resultou em alterações microscópicas em tecidos oculares outros que não o local da aplicação.

\section{Agradecimentos}

Ao Prof. Dr. Ricardo Luis Smith, do Depto. de Morfologia da UNIFESP e ao Hospital Veterinário da Universidade de Cuiabá UNIC/MT.

\section{Referências}

BISTNER, S. et al. Diseases of the Uveal Tract (Part II). In: Glaze, M.B. The Compendium Collection: Ophthalmology in Small Animal Practice. 2. ed. New Jersey: Veterinary Learning Systems. 1996, p. 169-176.

BLAIR, M.J. et al. Subconjunctivally implanted micro-osmotic pumps for continuous ocular treatment in horses. American Journal of Veterinary Research. v. 60, n. 9, Sept. 1999.

BOOTHE, D. M. Prostaglandins: Phisiology and clinical implications. The Compendium on Continuing Education for the Practicing Veterinarian. v. 6, n. 11, p. 1010-1021. Nov.1984.

. Controlling inflammation with nonsteroidal anti-inflammatory drugs. Veterinary Medicine, p. 875-883. Sept.1989. Symposium on Practical Drug Therapy in Pets.

COLLINS, B. K.; MOORE, C. P. Diseases and Surgery of the canine anterior uvea. In: GELLAT, K. N. Veterinary Ophthalmology. Philadelphia: Lippincott Williams \& Wilkins. $3^{\text {rd }}$. ed. 1999. p. 755-795.

CONLON, P.D. Nonsteroidal drugs used in the treatment of inflammation. Veterinary Clinics of North America: Small Animal Practice. v. 18, n. 6, p. 1115-1131. 1988.

COTRAN, R. S.; KUMAR, V.; ROBBINS, S. L. Acute and Chronic Inflammation. In: Pathologic Basis of Disease. Philadelphia: W.B. Saunders. 6th ed.1999. ch. 3, p. 50-88.

CRUZ, M. L. et al. Avaliação dos efeitos gastrointestinais do Flunixin Meglumine em cães. A Hora Veterinária. ano 18, n. 103, p. 33-38, 1998.

DONZIS, P. B.; MONDINO, B. J. Management of Noninfectious Corneal Ulcers. Survey of Ophthalmology. v. 32, n. 2, p. 94-108. March.1987.

DZIEZYC, J. et al. Effects of lipoxygenase inhibitors in a model of lensinduced uveitis in dogs. American Journal of Veterinary Research, v. 50, n. 11, p. 1877-1882, 1989.

ELWOOD, C. et al. (Letters). Renal failure after flunixin meglumine administration. Veterinary Record, v. 27, p. 582-583. 1992.
FANTONI, D. T.; CORTOPASSI, S. R. G.; BERNARDI, M. M. Anestésicos Intravenosos e outros parenterais. In: SPINOSA, H. S; GORNIÁK, S. L.; BERNARDI, M. M. Farmacologia Aplicada à Medicina Veterinária. 3. ed. Rio de Janeiro: Guanabara Koogan. 2002, Cap. 11, p. 117-128.

FORSYTH, W. G.; GUILFORD, W. G.; PFEIFFER, D. U. Effect of NSAID administration on creatinine clearance in healthy dogs undergoing anaesthesia and surgery. Journal of Small Animal Practice, v. 41, p. 547-550. 2000.

HALL, D. W. R.; BONTA, I. L. Prostaglandins and ocular inflammation. Documenta Ophthalmologica, v. 44, n. 2, p. 421-434. 1977.

HARDIE, E. M.; HARDEE, G. E.; RAWLINGS, C. A. Pharmacokinetics of flunixin meglumine in dogs. American Journal of Veterinary Research, v. 46 , n. 1, p. 235-237, 1985.

HENDRIX, D. V. Corneal stromal abscesses in the horse: a review of 24 cases. Equine Veterinary Journal, v. 27, n. 6, p. 440-447. 1995.

HIGGINS, A. J.; LEES, P. The acute inflammatory process, arachidonic acid metabolism and the mode of action of anti-inflammatory drugs. Equine Veterinary Journal, v. 16, n. 3, p. 163-175. 1984.

KING, C. S.; MILLER, R. T. Fatal perforating intestinal ulceration attributable to flunixin meglumine overdose in rats. Laboratory Animal Science, v. 47, n. 2, p. 205-208. 1997.

. A. Effects of Flunixin Meglumine and Dexamethasone on aqueous protein values after intraocular surgery in the dog. American Journal of Veterinary Research, v. 48, n. 3, p. 420-422, 1987a.

. Ocular use of anti-inflammatory drugs in companion animals.

The Compendium on Continuing Education for the Practicing Veterinarian, v.9, n.11, p. 1085-1096. 1987b.

.. Ophthalmic usage on nonsteroidal anti-inflammatory agents. In: Current Veterinary Therapy: Small Animal Practice. Philadelphia: W.B. Saunders, 1989. p. 642-644.

LAUS, J.L. et al. Terapia anti-inflamatória na cirurgia da catarata pela facoemulsificação em cães. A Hora Veterinária, v. 21.n. 121, p. 19-23, maio/jun. 2001. 
LEES, P.; HIGGINS, A. J. Flunixin inhibits prostaglandin $E_{2}$ production in equine inflammation. Research in Veterinary Science, v. 37, n. 3, p.347349. 1984.

LOBETTI, R. G.; JOUBERT, K. E. Effect of administration of nonsteroidal anti-inflammatory drugs before surgery on renal function in clinically normal dogs. American Journal Veterinary Research, v. 61, n. 12, p. 1501-1507.2000.

MATHEWS, K. A. Nonsteroidal antiinflammatory analgesics - Indications and contraindications for Pain Management in Dogs and Cats. Veterinary Clinics of North America - Small Animal Practice, v. 3, n. 4, p. 783804, 2000.

MATHIS, G. A. Clinical Ophthalmic Pharmacology and Therapeutics. In: GELLAT, K.N. Veterinary Ophthalmology. Philadelphia: Lippincott Williams \& Wilkins. $3^{\text {rd }}$ ed. 1999. Ch. 8, p. 291-297.

McNEIL, P.E. Acute tubulo-intersticial nephritis in a dog after halothane anaesthesia and administration of flunixin meglumine and trimethoprim - sulphadiazine. Veterinary Record, v. 131, p. 148-151. 1992.

MEALEY, K. Systemic absorption of topically administered drugs. The Compendium on Continuing Education for the Practicing Veterinarian, v. 22, n. 7, p. 649-655, 2000.

MILLICHAMP, N. J.; DZIEZYC, J. Comparison of Flunixin Meglumine and Flurbiprofen for control of ocular irritative response in dogs. American Journal of Veterinary Research, v. 52, n. 9, p. 1452-1455, 1991.

MILLICHAMP,N. J. et al. Acute effects of anti-inflammatory drugs on neodymium:yttrium aluminum garnet laser- induced uveitis in dogs. American Journal of Veterinary Research, v. 52, n. 8, 1279-1284, 1991.
PEIFFER, R. L. Ocular Immunology and Mechanisms of Ocular Inflammation. Veterinary Clinics of North America: Small Animal Practice, v. 10, n. 2, p. 281-301, 1980.

REGNIER, A. Antimicrobials, anti-inflammatory agents, and antiglaucoma drugs. In: GELLAT, K. N. Veterinary Ophthalmology. Philadelphia: Lippincott Williams \& Wilkins. $3^{\text {rd }}$ ed. 1999, Ch. 8, p. 297-336.

SLATTER, D. Fundamentals of Veterinary Ophthalmology. Philadelphia: W.B. Saunders Company. $3^{\text {rd }}$ ed. 2001, 640p.

SHIELS, I. A; SANDERSON, S. D.; TAYLOR, S. M. Arterially perfused eye model of uveitis. Australian Veterinary Journal, v. 77, n. 2; p.100104, 1999.

STADES, F.C. et al. Diagnósticos e Terapêuticas para Doenças Oculares. In: _. Fundamentos de oftalmologia veterinária. São Paulo: Manole. 1999, Cap. 3, p. 22-30.

TASAKA, A. C. Antiinflamatórios não esteroidais. In: SPINOSA, H. S; GORNIÁK, S. L.; BERNARDI, M. M. Farmacologia aplicada à medicina veterinária. 3ed. Rio de Janeiro: Guanabara Koogan, 2000, cap.21, p. 224-239.

VONDERHAAR, M. A.; SALISBURY, S. K. Gastroduodenal ulceration associated with flunixin meglumine administration in three dogs. Journal of American Veterinary Medicine Association, v. 203, n. 1, jul. 1993.

WAGONER, M. D. Chemical injuries of the eye: current concepts in pathophisiology and therapy. Survey of Ophthalmology, v. 41, n. 4, Jan-Feb 1997.

WARD, D. A.; CLARK, E. S. Ocular Pharmacology. The Veterinary Clinics of North America: Food Animal Practice, v. 7, n. 3, p. 779-790, 1991. 